\title{
Diagnostic STEM-EELS Analysis of Cathodes Degradation in Lithium-Ion Secondary Batteries
}

\author{
S. Muto, ${ }^{*}$ K. Tatsumi, ${ }^{*}$ Y. Kojima, ${ }^{*}$ H. Kondo, ${ }^{* *}$ T. Sasaki, ${ }^{* *}$ K. Horibuchi** and Y. Ukyo** \\ * Graduate School of Engineering, Nagoya University, Nagoya 464-8603, Japan \\ ** Toyota Central R\&D Laboratories, Nagokute 480-1192, Japan
}

Rechargeable lithium ion batteries are now considered for the automotive applications because of their high energy densities and voltages. To improve battery performance, it is particularly necessary to provide insight into the degradation processes in cathodes at elevated temperature. TEM and related techniques can surely serve this field at nanometer or sub-nanometer resolution.

We have examined various types of cathodes before and after charge-discharge cycling tests in the test cells, as large areas as a few to tens micrometers of which were thinned by FIB. We applied a multivariate curve resolution (MCR) technique to spectrum imaging (SI) datasets obtained by STEM-EELS, to resolve the overlapping unknown spectral components, thereby visualizing the spatial distribution of each different chemical state on a relative concentration map, as shown in Fig. $1[1]$.

NCA ( $\left.\mathrm{LiNi}_{0.8} \mathrm{Co}_{0.15} \mathrm{Al}_{0.05} \mathrm{O}_{2}\right)$ cathode: The method successfully revealed that a NiO-like phase was distributed near the surface of NCA particles after the cycling test at elevated temperature $\left(70-80^{\circ} \mathrm{C}\right)$, and that device degradation could be largely explained by the evolution of this NiO-like phase, particularly near the NCA particle surface [2,3], as shown in Fig. 2. Furthermore, the analysis of Li $\mathrm{K}$ ELNES revealed that the initial conditioning formed a thin $\mathrm{Li}_{x} \mathrm{PO}_{y} \mathrm{~F}_{z}$ layer along the active material surface due to the reaction with the $\mathrm{LiPF}_{6}$ electrolyte [4], and lithium fell into the Ni site in the NiO-like degraded phase [3].

Mg-doped $\mathrm{NCA}\left(\mathrm{LiNi}_{0.75} \mathrm{Co}_{0.15} \mathrm{Al}_{0.05} \mathrm{Mg} \mathrm{g}_{0.05} \mathrm{O}\right)$ cathode: To improve device stability, we proposed $\mathrm{Mg}$-doped-NCA as a cathode material. This actually suppressed capacity fading and resistance increase during cycling at $60-70^{\circ} \mathrm{C}$. We examined the state of $\mathrm{Mg}$ in NCA and found in comparison with the aid of $a b$ initio calculations that $\mathrm{Mg}$ is mainly substituted at the $\mathrm{Ni}$ site, and that $\mathrm{Ni}-\mathrm{O}$ bonding around the dopant was significantly increased due to a greater degree of ionic bonding [5]. Spatial mapping of the Mg-doped NCA sample showed less degradation than the NCA cathode, after cycling at $70{ }^{\circ} \mathrm{C}$, and the degraded regions of the Mg-doped NCA cathode are in a spinel phase, rather than the NiO-like phase seen in undoped NCA, as shown in Fig. 3 [6].

Two-phase morphology of $\mathrm{LiFePO}_{4} / \mathrm{FePO}_{4}$ : Unlike the layered oxides such as NCA, there have been a number of models proposed for lithium ion transport associated with the active material particle morphologies, depending on the various preparative methods of $\mathrm{LiFePO}_{4}$. We found coreshell morphologies in the particle larger than $100 \mathrm{~nm}$ in $50 \%$ SOC (state-of-charge) prepared by starting from the fully charged and fully discharged states, where the core-shell relation is inverted each other, as shown in Fig. 4. Plausible models for the charge/discharge process are discussed.

References

[1] S. Muto et al., Mater. Trans. 50 (2009) 964.

[2] S. Muto et al., J. Electrochem. Soc. 156 (2009) A371; 
[3] Y. Kojima et al., J. Power Sources, submitted (2010).

[4] S. Muto et al., Electrochem. Solid-State Lett.13 (2010) A115.

[5] K. Tatsumi et al., Phys. Rev. B 78 (2008) 045108.

[6] H. Kondo et al., J. Power Sources, 174 (2007) 1131; S. Muto et al., Phys. Chem. Chem. Phys., submitted (2011).

[7] This research was supported by a Grant-in-Aid for Scientific Research (KAKENHI) in Priority Area (\#474) “Atomic Scale Modification” from MEXT, Japan.

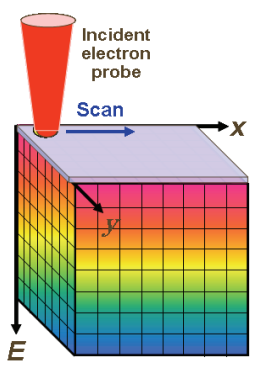

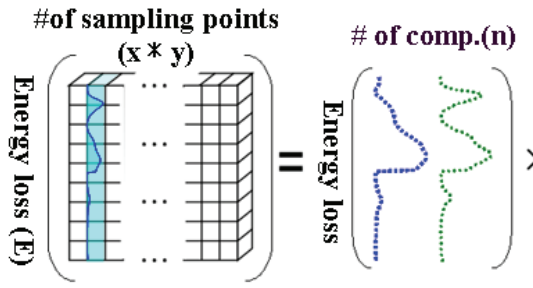

Data matrix
Comp. spectra

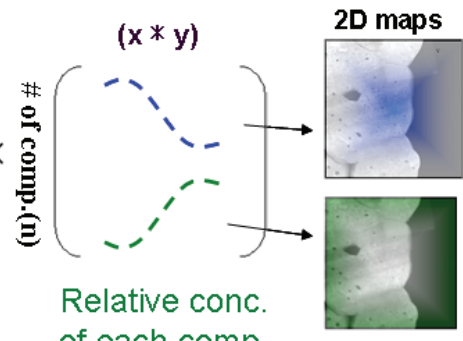

of each comp.

FIG. 1. Schematic diagram of STEM-EELS spectrum imaging (left), combined with MCR technique (right: two-component case) [1].
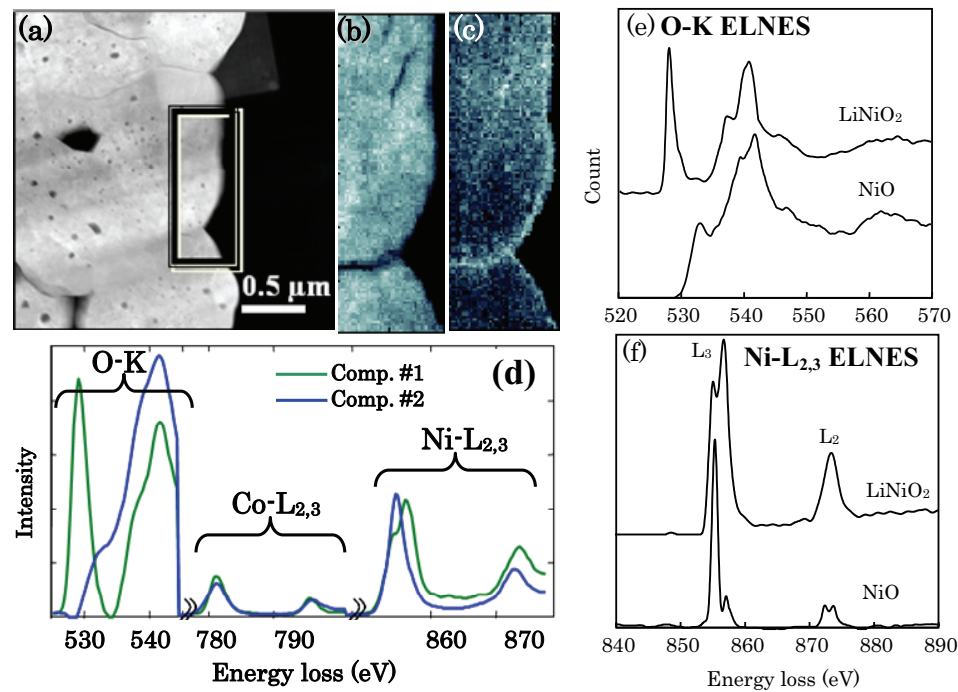

FIG. 2. (a)ADF-STEM image of NCA cathode. The framed area was scanned for spectrum imaging. (b),(c)Spatial distribution maps of component 1 and 2, respectively. (d)Resolved spectral components: compared with reference (e) and (f), Component 2 can be identified to be $\mathrm{NiO}$.
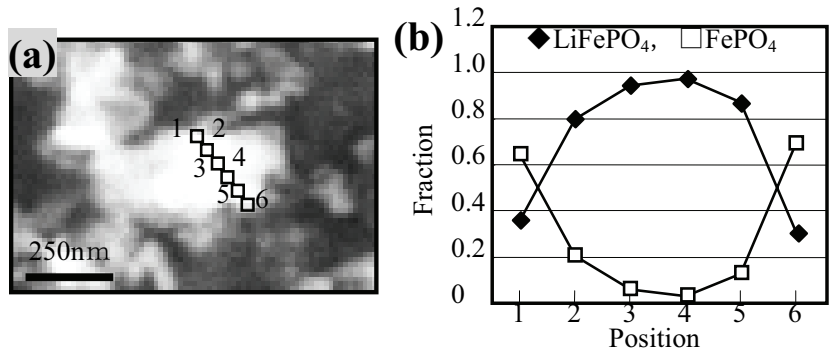

FIG. 4. (a)ADF-STEM image of $\mathrm{LiFePO}_{4} /$ $\mathrm{FePO}_{4}$ particles (SOC50\%, lithiated from SOC0\%). (b) Fractions of $\mathrm{LiFePO}_{4}$ and $\mathrm{FePO}_{4}$ at the positions indicated in (a), suggesting the core-shell structure. The core-shell relation is inverted in the delithiated SOC50\% sample. 